\title{
Diagnostic Procedure and Therapeutic Approaches in Nonconvulsive Status Epilepticus (NCSE)
}

\author{
Zoryana Lebedyn
}

\begin{abstract}
Nonconvulsive epileptic seizures are major type of seizures of all patients with predominantly focal epilepsy. However, this problem is still insufficiently studied, the literature presents a few data on the diagnosis and treatment of nonconvulsive status epilepticus (NCSE). NCSE is a prolonged seizure, not accompanied by severe motor presentation. Such attacks occur much more often than previously thought. NCSE is divided into focal NCSE (complex partial status epilepticus), and generalized NCSE, often referred as absence status. The absence status divides into a typical absence status (presents with suppression of consciousness of various depths, myoclonic twitching of the eyelids, impoverishment of speech production and hallucinations), atypical absence status (presents with retardation of mental processes and eyelid myoclonus) and late absence status (long-term disorientation accompanied by cognitive deficiency and presents mainly in elderly patients). According to the available sources, more than $30 \%$ of patients in the intensive care units with impaired consciousness of unclear genesis are patients with NCSE. Use of antiepileptic drugs for intravenous administration opens better opportunities in the treatment of patients with status epilepticus and NCSE.
\end{abstract}

Index Terms - nonconvulsive status epilepticus, NCSE, epilepsy, status epilepticus, neurology, neuropsychiatry.

\section{INTRODUCTION}

Status epilepticus (SE) is a pathological condition characterized by epileptic seizures lasting more than 5 minutes or recurrent seizures, in the interval between which the functions of the central nervous system are not fully restored [1]. Nonconvulsive status epilepticus (NCSE) is a prolonged seizure that is not accompanied by severe motor manifestations [2].

The separation of the NCSE from the SE is fundamentally important for two reasons. First of all, it is due to the difficulties of clinical diagnosis, as convulsive manifestations are absent and complex partial seizures during the NCSE may not be accompanied by changes in epileptic activity on the EEG. Secondly, in children, especially at an early age, it is often impossible to identify the NCSE. In addition, the NCSE is a condition that significantly impairs the patient's health, but is often interrupted and reversed [2], [3].

Criteria for diagnosis and treatment still remain controversial. Usually, the NCSE is manifested by ictal suppression of consciousness, twitching of the face or limbs, deviation of the eyes or head, automatisms, changes in behavior. The clinical polymorphism requires recording and identification of the attack on the EEG, although the determination of NCSE by EEG could be compromised.

In intensive care units for neurological patients (neurology ICU), up to $34 \%$ of patients who undergo EEG have nonconvulsive seizures, and $76 \%$ of these seizures have NCSE [4]. This confirms the relevance and importance of the study of this problem. Even after excluding of all patients with a history of seizures, up to $8 \%$ of patients in a coma have NCSE [5]. According to some authors, NCSE has been described in $27 \%$ of patients with altered consciousness in $48 \%$ of patients after generalized status epilepticus, in $22 \%$ of patients with severe brain damage of traumatic origin, in $6 \%$ of patients with ischemic stroke and $28 \%$ of patients with intracerebral haemorrhage [6]-[10]. It is especially important to diagnose such patients as early as possible, as factors associated with seizure activity (changes in cerebral metabolic activity, mismatch of neuronal energy consumption and energy supply) can dramatically worsen the further prognosis for the patient in the intensive care unit.

\section{CliniCAL PRESENTATION AND DIAGNOSIS OF NCSE}

In general, NCSE is traditionally divided into focal NCSE (complex partial NCSE) and generalized NCSE, often referred to as absence status. The generalized type is characterized by confusion of consciousness accompanied by blinking or twitching of the facial muscles, lasting from several hours to several days, which is accompanied by the registration of the slow spike-and-wave discharge on the EEG. At the same time, the absence status itself may be divided into a typical absence status (with presentation of suppression of consciousness of various depths, myoclonic twitching of the eyelids, impoverishment of speech production and hallucination), atypical absence status (which presents with slowing of mental processes, myoclonus) and late absence status (with presentation of long-term disorientation accompanied by a cognitive deficit and mainly observed in elderly patients).

Complex partial NCSE includes prolonged or repeated partial seizures (with a focal onset) and manifests itself as a prolonged epileptic twilight state with fluctuations in the degree of severity and suppression. Therefore, the overlap of syndromes is possible. Other comatose cases where rapidly occurring rhythmic epileptiform discharges on the EEG may have a clear recorded status and can be attributed to a similar diagnostic category. NCSE most often develops after the relief of seizures along with the presentation of neurological 
dysfunction, despite adequate treatment.

Absence NCSE is generally characterized by the absence of signs of focal epilepsy and is accompanied by the registration of rapid generalized epileptiform discharges with a frequency of $3 \mathrm{~Hz}$ on the EEG. The clinical features have been recognized [11]:

1. The state confusion of consciousness during wakefulness, accompanied by blinking or periodically arising myoclonus of the eyelids;

2. May begin or end with clinical presentation of generalized seizures;

3. Duration can last few days;

4. Minimal focal features and generalized discharges on EEG;

5. Absences, myoclonic, or convulsive seizures in the history;

6. Typically provoking events: withdrawal of alcohol, benzodiazepines or other drugs, generalized seizures, infection, trauma, metabolic disorders.

Often, in such cases, patient suffers extremely prolonged absence seizures. In some studies, frequent episodes have been described in elderly patients without prior epilepsy (de novo status epilepticus) in combination with the withdrawal of benzodiazepines used to relieve anxiety or as a hypnotic [12]. In addition to absences, several types of generalized seizures are distinguished, which, with the proper duration, may correspond to the structure of status epilepticus [13]. Such conditions include myoclonic status, tonic, clonic, and tonic-clonic seizures, each of the presented types of which is accompanied by a pronounced motor manifestation without being non-convulsive.

Complex partial status epilepticus is a variant of NCSE with an assumed focal onset, the clinical symptoms of which are most often represented by the following signs:

1. The degree of clouding of consciousness to the level of unresponsiveness;

2. Epileptic twilight states;

3. Recurrent or prolonged complex partial seizures;

4. Observed or proposed focal onset, often temporal or frontal localization;

5. Positive history of previous epilepsy and vascular pathology in elderly patients;

6. May last for months with possibility of a recurrent flow;

7. May cause severe amnesia that develops later.

Complex partial status epilepticus has been mentioned in the literature to date somewhat less often than absence status epilepticus, possibly due to strict definitions of this condition. Clinical manifestation includes epileptic twilight consciousness along with a significant decrease in activity, confusion, and, in particular, fluctuations in the behavioral activities [14]. Oral and/or hand automatisms can be noted periodically. In the case of complex partial status epilepticus, repeated complex partial seizures are observed along with ongoing seizure activity. Due to their association with vascular pathology or focal epilepsy, which precedes the current onset of complex partial status epilepticus, they are considered to be somewhere more difficult to treat, more prone to a truly refractory course of status epilepticus than absence status epilepticus, along with a greater tendency to recurrence and continued course.

The classification of NCSE is significantly complicated by the similarity of the characteristics of absence status epilepticus and complex partial status epilepticus, what may explain the prevalence of reports describing absence status epilepticus, while complex partial seizures in clinical practice of adult patients are generally more common. In both cases, states with confusion or other changes in the cognitive functioning, accompanied by minimal motor activity, usually along with the absence of systemic somatic pathology, are included. Information about positive history regarding primary generalized epilepsy, as well as rapidly emerging rhythmic generalized discharges on the EEG indicate absence epilepsy, while focal seizures or other focal neurological pathology and fluctuating course of the disease course in certain way indirectly indicates complex partial status epilepticus. However, in certain cases, the seizure may have a focal onset and undergo rapid generalization.

Cases describing atypical absence status epilepticus, the distinctive characteristics of which were mentioned above, may in fact describe complex partial seizures with generalization and prolongation. This concept is based on the results of a search for correlations between clinical presentation and EEG data.

While studied 32 patients with NCSE, 14 of whom had focal EEG changes (complex partial status epilepticus was suggested), while 18 had generalized discharges, 6 of whom had primary generalized epilepsy. At the same time, patients could not be confidently assigned to any of the groups on the basis of clinical manifestations alone [12].

In patients in a state of lethargy, stupor, or even coma, EEG often reveals rapidly arising rhythmic continuous discharges, accompanied or not accompanied by clinical manifestations of seizures in the period following clinically expressed convulsions or convulsive status epilepticus. Rarely, EEG discharges or seizures are the only problems; many of the patients suffer from serious cerebrovascular pathology, serious mental disorder or encephalopathies of various origins (toxic/dysmetabolic, or of mixed etiology) [15]-[18]. These patients can be attributed to the group of patients with electrographic status epilepticus or epileptic encephalopathy.

\section{NEUROPSYCHIATRY OF NCSE}

Bipolar disorder is indeed a common mental disorder, with $1-3 \%$ of people having it. [19]-[21]. For more than twothirds of patients with uncomplicated bipolar disorder, lithium is effective in softening mood swings but not affecting normal mood. However, even in the therapeutic range lithium treatment may trigger NCSE onset [22].

Depressive disorders are characterized by a rather pronounced and persistent sadness, as a result of which a person's activity is disrupted and interest in life decreases [23]-[26]. The main indication for ECT is major depressive disorder depression that is resistant to antidepressant therapy. NCSE is a serious, uncommon under-reported side-effect associated with ECT that should be mentioned [27].

Mental disorders occur in 30-40\% of people with epilepsy, although when epilepsy is combined with other severe neurological disorders, this figure reaches $60 \%$ [28]. The prevalence of mental disorders is also increased with temporal lobe epilepsy. NCSE clinical features are subtle and non-specific and, consequently, they are generally not 
diagnosed and confused with behavioral or psychiatric disorders [29]. It remains a challenge to characterize neurobiological changes that contribute to the genesis or maintenance of both conditions, with a clear need for further investigation. They can be due to various reasons. Firstly, a number of diseases have been identified in which the brain damage underlying epilepsy also causes the development of mental disorders. Such diseases include, for example, tumors of the frontal lobe and Alzheimer's disease (the most common form of acquired dementia (dementia) with onset in old age).

Attention deficit hyperactivity disorder (ADHD) refers to disorders of the nervous system [30]-[32]. Neurological disorders are based on prerequisites of a neurological nature that appear in early childhood, usually before entering school, and that are detrimental to personal, social, academic and / or professional growth. They are usually associated with difficulties in acquiring, maintaining, or applying special skills or sets of information. Neurodevelopmental disorders can include decreased attention, memory, perception, speech, inability to solve problems, or impaired social interaction. Patients with ADHD often have comorbidities that create additional diagnostic and therapeutic problems and lead to an even greater decrease in the quality of life of patients [33]. ADHD is often combined with epilepsy. Moreover, compared with the general population, not only is ADHD more common in epilepsy, but epilepsy is more common in the ADHD [33]. ADHD patient may be at risk for NCSE.

Pathological gambling is an urgent medical and social problem of modern society, which is associated with high prevalence rates and comorbidity with social problems and mental disorders [34]. Gambling has been reported with NCSE patient [35].

\section{TREATMENT OF NCSE}

For several decades, one of the most effective drugs used both in combination with other anticonvulsants and in monotherapy, prescribed for both partial and generalized attacks is sodium valproate [36]. The effectiveness of this medication in its application is associated with the effect on the GABA system by enzymatic increase in the quantitative presence of GABA at the postsynaptic level in combination with blocking the catabolic processes of GABA. In addition, there are other beneficial effects of the use of valproic acid on the hormonal system. The study of the mechanism of action of valproic acid made it possible to identify a number of forms of epilepsy, in which the preparations of valproic acid show the expected effectiveness. Considering the aspect of non-convulsive epileptic seizures and NCSE, taking into account the variety of clinical and subclinical manifestations of the seizures observed in this case, it is required that the drugs used to relieve the status have efficacy in relation to both partial, symptomatically caused epilepsies, and in relation to absence forms.

Sodium valproate is available in the form of $500 \mathrm{mg}$ of active substance in $5 \mathrm{ml}$ of solution in vials. In ICU conditions, where there is a dynamic observation and correction of therapy for patients diagnosed with status epilepticus, it is extremely important to achieve an adequate concentration for a short period of time and maintain it through continued infusion. Valproic acid at a concentration of $50-100 \mathrm{mg}$ binds to plasma proteins by $80-85 \%$, with a plasma concentration of less than $50 \mathrm{mg}$ by $90-95 \%$. The drug undergoes glucuronidation and oxidation in the liver, while the excretion of $1-3 \%$ of the dose of valproic acid and its metabolites is performed by the kidneys, a small amount is excreted in the feces and exhaled air. The half-life for monotherapy with valproic acid in healthy volunteers is from 8 to 20 hours, while it should be remembered that with multiresistant, difficult to treat forms of epilepsy, requiring a combination of several antiepileptic drugs, the half-life may increase in patients with hepatic pathology, or decrease in patients receiving anti-epileptic drugs (AEDs) [36]. Based on the data of the International standards for the relief of status epilepticus, it can be noted that valproic acid has a more favorable pharmacological profile than other anticonvulsants, while valproic acid preparations can be administered intravenously, without significantly affecting respiratory function and hemodynamics [37].

When sodium valproate administered intravenously at a dose of $15-16 \mathrm{mg} / \mathrm{kg}$ of body weight, followed by support in the form of infusion therapy at a dose of $0.5-4 \mathrm{mg} / \mathrm{kg}$ of body weight $/ \mathrm{h}$ for a period of 2 hours to 10 days [38]. None of the patients had the cardiovascular side effects, while drug remission was established in $29(85.3 \%)$ patients with serial seizures and in $27(77.1 \%)$ patients with status epilepticus. The most pronounced beneficial effect from the use of valproate was seen in patients with complex partial status epilepticus, while in patients with atypical absence status epilepticus, the effectiveness of therapy was the least. Over the past 20-30 years, numerous studies have established high efficacy and good tolerance of intravenous valproate for the relief of status epilepticus in adults and children.

In situations in which the attending physician is faced with the presence of status epilepticus in the patient with the refractory to the treatment, a medication is required with the mechanism of action of which would not repeat those of the drugs already used without obtaining an effect, or additionally potentiated the effect of the drugs used. Levetiracetam has unique properties that determine its pharmacokinetics and dose titration. This medication affects the intraneuronal concentration of calcium ions, partially slowing down their passage through the $\mathrm{N}$-type channels and thereby reducing the release of calcium from intraneuronal stores [39]. One of the proposed mechanisms is based on the proven binding to the SV2A synaptic vesicle glycoprotein, which is contained in the gray matter of the brain and spinal cord. It is believed that in this way the anticonvulsant effect is realized, which is expressed in counteracting the hypersynchronization of neuronal activity. Levetiracetam also acts on GABA and glycine receptors, modulating these receptors through various endogenous agents. The qualitative difference between this drug is the possibility of prescribing in a dose corresponding to its initial therapeutic concentration in blood plasma. Other antiepileptic drugs require, in order to avoid the occurrence of side effects, a gradual increase in the dose to the therapeutic dose over days, weeks and even months, which is unacceptable in the ICU, where patients with status epilepticus or serial seizures are treated. In addition, due to the high level of absorption of a linear nature, the bioavailability of the drug, approaching $100 \%$, it becomes possible such an important measure as calculating the plasma 
concentration in the blood depending on the dose used [40]. At the same time, therapeutic concentration, the concentration of the drug in the blood plasma, leading to the development of a full therapeutic effect, becomes achievable in less than 1 hour. At the same time, daily oral effective doses for the treatment of refractory status epilepticus in the cases described in the literature vary from 500 to $9000 \mathrm{mg}$. The emergence of the intravenous form of levetiracetam opens up new prospects for its use. One of the most appropriate options is its initial intravenous administration followed by supportive oral therapy. In addition to treating refractory status epilepticus, intravenous levetiracetam is recommended as a maintenance treatment for a patient with status epilepticus after initial successful therapy with diazepam (often used as the drug of choice in ICU settings), providing additional safety in relation to the suppression of vital functions, which is observed with repeated administration of benzodiazepines, therefore helping to avoid undesirable effects inherent in benzodiazepine drugs [41][43].

It is widely believed that various medical procedures, including dental, are associated with great risks in patients with epilepsy [44], [45]. The onset of seizures is possible during or immediately after the above procedures due to the potential for interactions between anesthetics and AEDs. There are simple precautions to take before undertaking medical intervention in patients with epilepsy. First, seizure triggering factors should be avoided. Due to the fact that skipping the drug is one of the most common causes of seizures, the doctor must be sure that the patient continues to take the AED regularly. Despite the fact that before most dental and surgical procedures, one should not eat or drink for at least 8 hours, the patient should take the medicine with several sips of water. Insomnia, which often occurs in patients on the eve of medical or dental interventions, is also a frequent trigger of seizures and should be avoided, for example, by giving the patient low doses of benzodiazepines.

In the line of antiepileptic drugs, the drug lacosamide is also available. Lacosamide is a modified amino acid exhibiting the properties of an anticonvulsant with a fundamentally new mechanism of action [39]. Unlike other modulators of sodium channels (carbamazepine, lamotrigine, phenytoin), lacosamide selectively increases the slow inactivation of sodium channels, which leads to stabilization of hyperexcitable neuronal membranes and slowing reexcitation of neurons. It is known that in patients with epilepsy, the expression of the CRMP-2 protein is impaired, and lacosamide, presumably, binds to this protein. CRMP-2 is involved in the process of neuronal differentiation and in the control of axonal growth [39], [46]. As noted above, the choice of an antiepileptic drug as an additional drug in a duo therapy or polypharmacy primarily depends on its mechanism of action, profile of side effects and clinical efficacy. Possible reasons for the failure of antiepileptic therapy include intolerable side effects prompting the physician to limit themselves to low doses of AED, as well as insufficient clinical efficacy. In this regard, the emergence of a new AED always raises questions about the features of its application and its possible place in the treatment of epilepsy.

The recommended starting dose of lacosamide is 50-100 $\mathrm{mg}$ with an increase of $100 \mathrm{mg}$ per week to a therapeutic dose of $200 \mathrm{mg}$. Treatment can be started with a tablet or intravenous form of the drug. In the case of insufficient effectiveness, increment of the dose of lacosamide to $400 \mathrm{mg}$ per day, divided into two doses is required. Lacosamide for intravenous injection is also used twice a day. Intravenous administration of lacosamide should be performed not faster than within 15 minutes, depending on tolerance. For elderly patients, dose adjustment or reduction is usually not required. The drug lacosamide is easy to use, food intake does not change the percentage and rate of absorption of the drug [39], [46]. Plasma concentration is directly proportional to the dose taken, which, as noted in the description of levetiracetam, is of great importance in calculating the plasma concentration of the drug. The maximum concentration of lacosamide is reached within 1-4 hours after oral administration, the halflife is about 13 hours and does not depend on the dose or duration of lacosamide use [39], [46]. Lacosamide weakly binds to plasma proteins and has a low potential for pharmacokinetic drug interactions, which makes it the most valuable and promising as an additional drug in duo therapy and polypharmacy.

Lacosamide has been shown to be highly effective compared to placebo. The percentage of patients who completed the study was at a fairly high level, averaging over $70 \%$. According to the research results, it was concluded that lacosamide is well-tolerated [47], [48]. Most of the side effects were dose dependent and occurred during the titration phase of the lacosamide dose. Side effects associated with the activity of the central nervous system and gastrointestinal functioning, but their severity were insignificant and did not require discontinuation of the drug. Dose-dependent side effects that appeared when taking lacosamide at a dose of 400 and $600 \mathrm{mg}$ per day included: impaired coordination, ataxia, nausea, vomiting, nystagmus, in some cases, causing early withdrawal of patients from the study. Very favorable is the fact that there are no reports of skin reactions and hyponatremia. There were no reports of impaired cognitive functions in the form of slowing of thinking, deterioration in the perception of new information, which is very important in working patients and elderly patients. No effect on body weight was found, which is a common reason for the discontinuation of a number of antiepileptic drugs. As a result of data analysis, it was shown that lacosamide does not change the plasma concentration of co-prescribed AEDs, such as carbamazepine, valproate, topiramate, phenytoin, oxcarbazepine, lamotrigine and levetiracetam. There also was no change in the concentration of lacosamide with the combined use of the above-mentioned AEDs. The greatest effectiveness of lacosamide was shown in combination with valproate, levetiracetam and topiramate [46]-[48].

\section{CONCLUSIONS}

The frequency of occurrence and the peculiarity of the clinical presentation and the disease course of NCSE encourage researchers and practicing physicians (neurologists, ICU specialists and psychiatrists) to continue search of effective approaches in diagnoses and treatment of NCSE and also determine the importance of developing drugs for the treatment of patients suffering from refractory to treatment forms of epilepsy and seizure disorders. 


\section{REFERENCES}

[1] Sirven, J. I., \& Waterhouse, E. (2003). Management of status epilepticus. American family physician, 68(3), 469-476.

[2] Towne, A. R., Waterhouse, E. J., Boggs, J. G., Garnett, L. K., Brown, A. J., Smith, J., \& DeLorenzo, R. J. (2000). Prevalence of nonconvulsive status epilepticus in comatose patients. Neurology, 54(2), 340-340.

[3] Jordan, K. G., \& Hirsch, L. J. (2006). In Nonconvulsive Status Epilepticus (NCSE), Treat to Burst-Suppression: Pro and Con. Epilepsia, 47, 41-45.

[4] Jordan, K. G. (1992). Nonconvulsive seizures (NCS) and nonconvulsive status epilepticus (NCSE) detected by continuous monitoring in the Neuro ICU (NICU-CEEG). Neurology, 42(13), 194.

[5] Towne, A. R., Waterhouse, E. J., Boggs, J. G., Garnett, L. K., Brown, A. J., Smith, J., \& DeLorenzo, R. J. (2000). Prevalence of nonconvulsive status epilepticus in comatose patients. Neurology, 54(2), 340-340

[6] Tsarkov, A., \& Petlovanyi, P. (2019). Neuropsychiatric Aspects of a Common Problem: Stroke. European Journal of Medical and Health Sciences, 1(3).

[7] DeLorenzo, R. J., Waterhouse, E. J., Towne, A. R., Boggs, J. G., Ko, D., DeLorenzo, G. A., ... \& Garnett, L. (1998). Persistent nonconvulsive status epilepticus after the control of convulsive status epilepticus. Epilepsia, 39(8), 833-840.

[8] Privitera, M., Hoffman, M., Moore, J. L., \& Jester, D. (1994). EEG detection of nontonic-clonic status epilepticus in patients with altered consciousness. Epilepsy research, 18(2), 155-166.

[9] Vespa, P. M., Nuwer, M. R., Nenov, V., Ronne-Engstrom, E., Hovda D. A., Bergsneider, M., ... \& Becker, D. P. (1999). Increased incidence and impact of nonconvulsive and convulsive seizures after traumatic brain injury as detected by continuous electroencephalographic monitoring. Journal of neurosurgery, 91(5), 750-760.

[10] Vespa, P. M., O'phelan, K., Shah, M., Mirabelli, J., Starkman, S. Kidwell, C., ... \& Martin, N. A. (2003). Acute seizures after intracerebral hemorrhage: a factor in progressive midline shift and outcome. Neurology, 60(9), 1441-1446.

[11] Schwab, R. S. (1953). A case of status epilepticus in petit mal. Electroencephalography and clinical neurophysiology, 5(3), 441

[12] Thomas, P., Beaumanoir, A., Genton, P., Dolisi, C., \& Chatel, M. (1992). 'De novo'absence status of late onset: Report of 11 cases. Neurology, 42(1), 104-104.

[13] Kimura, S., \& Kobayashi, T. (1996). Two patients with juvenile myoclonic epilepsy and nonconvulsive status epilepticus. Epilepsia, 37(3), 275-279.

[14] Engel, J., Ludwig, B. I., \& Fetell, M. (1978). Prolonged partial complex status epilepticus: EEG and behavioral observations. Neurology, 28(9), 863-863.

[15] Drislane, F. W., \& Schomer, D. L. (1994). Clinical implications of generalized electrographic status epilepticus. Epilepsy research, 19(2), 111-121.

[16] Tsarkov A, Msoni P and Petlovanyi P. (2020). Uncommon presentation: Folie à deux (Case study). World Journal of Advanced Research and Reviews, 6(3), 43-49.

[17] Anatolii, T., Patrick, M., \& Petro, P. (2020). Uncommon presentation: Folie à deux (Case study). World Journal of Advanced Research and Reviews, 6(3), 043-049.

[18] Petlovanyi, P., \& Tsarkov, A. Child Schizophrenia: Theory and Practice.

[19] Tsarkov, A., \& Petlovanyi, P. (2016). Bipolar Disorder in Child Psychiatric Practice: A Case Report. Medical Journal of Zambia, 43(1), 41-46.

[20] Tsarkov, A., \& Petlovanyi, P. (2016). Bipolar disorder in child psychiatric practice.

[21] Tsarkov, A., \& Petlovanyi, P. The Role of Lamotrigine in the Treatment of Bipolar Depression.

[22] Bellesi, M., Passamonti, L., Silvestrini, M., Bartolini, M., \& Provinciali, L. (2006). Non-convulsive status epilepticus during lithium treatment at therapeutic doses. Neurological sciences : official journal of the Italian Neurological Society and of the Italian Society of Clinical Neurophysiology, 26(6), 444-446.

[23] Lebedyn, Z. (2020). Antidepressants In General Practice And Psychiatry. European Journal of Medical and Health Sciences, 2(3). https://doi.org/10.24018/ejmed.2020.2.3.318

[24] Lebedyn, Z. (2019). The Use of Antidepressants by General Practitioners and Psychiatrists ( Personal Experience ).

[25] Petlovanyi, P., \& Tsarkov, A. (2017). Depressive Disorder in Child Psychiatric Practice: A Case Report. Health Press Zambia Bull, 1(5), 9.

[26] Tsarkov, A., \& Petlovanyi, P. Depressive Disorder in Child Psychiatric Practice: A. The Health Press, 9.
[27] Reeve-Johnson, L., Unwin, A., \& Mc Connell, H. (2014). Generalised Non-Convulsive Status Epilepticus (NCSE) following ElectroConvulsive Therapy. J Psychol Psychother, 4(138), 2161-0487.

[28] Krishnamoorthy, E. S., Trimble, M. R., \& Blumer, D. (2007). The classification of neuropsychiatric disorders in epilepsy: a proposal by the ILAE Commission on Psychobiology of Epilepsy. Epilepsy \& Behavior, 10(3), 349-353.

[29] López Arteaga, T., Amo, C., Serrano González, C., \& Huertas Sánchez, D. (2013). Nonconvulsive status epilepticus and psychotic symptoms: case report. Rivista di psichiatria, 48(3), 268-270.

[30] Petlovanyi P and Tsarkov A. (2020). Practical guide and some recommendations for the diagnosis and management of Attention deficit hyperactivity disorder (ADHD). World Journal of Advanced Research and Reviews, 6(3), 257-261.

[31] Petro, P., \& Anatolii, T. (2020). Practical guide and some recommendations for the diagnosis and management of Attention deficit hyperactivity disorder (ADHD). World Journal of Advanced Research and Reviews, 6(3), 257-261.

[32] Tsarkov, A., \& Petlovanyi, P. (2017). Omega-3 Fatty Acids as an Alternative Treatment for Children with Attention Deficit Hyperactivity Disorder. Imperial Journal of Interdisciplinary Research (IJIR), 3, 1378-1380.

[33] Cohen, R., Senecky, Y., Shuper, A., Inbar, D., Chodick, G., Shalev, V., \& Raz, R. (2013). Prevalence of epilepsy and attention-deficit hyperactivity (ADHD) disorder: a population-based study. Journal of child neurology, 28(1), 120-123.

[34] Tsarkov, A., \& Petlovanyi, P. (2017). Pathological Gambling: The Old Problem of the Modern World. Imperial Journal of Interdisciplinary Research, 3(8), 216-221.

[35] Storrier, S., \& Beran, R. G. (2014). Compulsive gambling possibly associated with antiepileptic medication. Epilepsy \& behavior case reports, 2, 15-16.

[36] Nevitt, S. J., Marson, A. G., Weston, J., \& Smith, C. T. (2018). Sodium valproate versus phenytoin monotherapy for epilepsy: an individua participant data review. Cochrane Database of Systematic Reviews, (8).

[37] Meierkord, H., Boon, P., Engelsen, B., Göcke, K., Shorvon, S., Tinuper, P., \& Holtkamp, M. (2010). EFNS guideline on the management of status epilepticus in adults. European journal of neurology, 17(3), 348-355.

[38] Peters, C. N., \& Pohlmann-Eden, B. (2005). Intravenous valproate as an innovative therapy in seizure emergency situations including status epilepticus - experience in 102 adult patients. Seizure, 14(3), 164-169.

[39] Stahl, S. M., \& Stahl, S. M. (2013). Stahl's essential psychopharmacology: neuroscientific basis and practical applications Cambridge university press.

[40] Fay, M. A., Sheth, R. D., \& Gidal, B. E. (2005). Oral absorption kinetics of levetiracetam: the effect of mixing with food or enteral nutrition formulas. Clinical therapeutics, 27(5), 594-598.

[41] Patel, N. C., Landan, I. R., Levin, J., Szaflarski, J., \& Wilner, A. N. (2006). The use of levetiracetam in refractory status epilepticus Seizure, 15(3), 137-141

[42] Pato-Pato, A., Rana-Martinez, N., Lorenzo-Gonzalez, J. R., \& CimasHernando, I. (2006). The value of an oral solution of levetiracetam in status epilepticus. Revista de neurologia, 42(12), 768-768

[43] Novy, J., Logroscino, G., \& Rossetti, A. O. (2010). Refractory status epilepticus: a prospective observational study. Epilepsia, 51(2), 251 256.

[44] Jacobsen, P. L., \& Eden, O. (2008). Epilepsy and the dental management of the epileptic patient. J Contemp Dent Pract, 9(1), 5462.

[45] Phiri, C., Tsarkov, A., Petlovanyi, P., \& Lingenda, G. Factors Contributing To Oral Diseases and Treatment Needs amongst Mental Patients at Chainama Hills College Hospital, Lusaka, Zambia.

[46] Jo, S., \& Bean, B. P. (2017). Lacosamide inhibition of Nav1. 7 voltagegated sodium channels: slow binding to fast-inactivated states. Molecular pharmacology, 91(4), 277-286.

[47] Brigo, F. (2017). Lacosamide monotherapy for newly diagnosed epilepsy. The Lancet Neurology, 16(1), 20-22.

[48] Abou Khaled, K., Khoury, J., Macaron, G., \& Richa, S. (2016). Forced normalization and psychosis following use of lacosamide. SeizureEuropean Journal of Epilepsy, 41, 96-99. 